\title{
A mindfulness-based program for improving quality of life among hematopoietic stem cell transplantation survivors: feasibility and preliminary findings
}

\author{
Paul Grossman • Diana Zwahlen • Jorg P. Halter • \\ Jakob R. Passweg • Claudia Steiner • Alexander Kiss
}

Received: 19 June 2014 / Accepted: 14 September 2014 / Published online: 10 October 2014

(C) Springer-Verlag Berlin Heidelberg 2014

\begin{abstract}
Health-related quality of life (HRQoL) is often substantially reduced among individuals who have undergone hematopoietic stem cell transplantation (HSCT), and incidences of depression, fatigue, and anxiety are elevated. We examined effects of a mindfulness-based intervention (MBI) compared to psycho-oncological telephone consultation upon HRQoL, depression, anxiety, and fatigue among HSCT survivors. Sixty-two medically stable patients participated in the study; they had completed HSCT $\geq 6$ months previously. Thirty-two were randomly assigned to intervention arms, and 30 were offered their treatment preference. MBI consisted of a structured 8-week program of mindfulness training. Assessments were made at baseline, post-intervention and 3 months follow-up. Primary outcome was HRQoL. Depression, fatigue, anxiety, and personal goal attainment were secondary measures. Non-completion of interventions was low in both groups (9\%, MBI; $7 \%$ control). Employing intention-totreat analysis, MBI, compared with comparison procedure, improved HRQoL and reduced depression and anxiety at post-intervention ( $p$ 's $<0.05$ ); Cohen's d effect sizes, 0.6-0.7; 3 -month follow-up benefits were modest. These findings demonstrate broad feasibility and acceptance of, as well as satisfaction and adherence with, a program of mindfulness training for HSCT survivors; findings also suggest improved HRQoL and well-being as a consequence of MBI.
\end{abstract}

P. Grossman $(\bowtie) \cdot$ D. Zwahlen $\cdot$ C. Steiner $\cdot$ A. Kiss Department of Psychosomatic Medicine, University Hospital Basel, Basel, Switzerland

e-mail: paul.grossman@usb.ch

J. P. Halter · J. R. Passweg

Department of Hematology, University Hospital Basel, Basel, Switzerland
Nevertheless, this is a preliminary study; a larger trial with more prolonged intervention phase is warranted.

Keywords Hematopoietic stem cell transplantation . Mindfulness · Quality of life · Depression · Anxiety · Fatigue

Substantial evidence indicates that mindfulness-based intervention (MBI) can improve HRQoL and other indices of wellbeing in cancer patients [1-5]. A pilot study with patients undergoing HSCT also suggested feasibility of MBI in this population [6]. Therefore, in this preliminary study aimed at both feasibility and possible HRQoL outcomes, we examined MBI among hematopoietic stem cell transplantation (HSCT) survivors.

Hematopoietic stem cell transplantation (HSCT) is a treatment used for a variety of life-threatening malignant hematological disorders, bone marrow deficiency diseases, and other illnesses. Despite evidence of 20-70 \% clinical cures of disorders (depending on disorder) and great advances over the years [7-12], this therapy is also associated with high and sustained morbidity and mortality during and after treatment, due to infection, immune suppression, organ failure, or recurrence of primary disease $[13,14]$. Additionally, there are a number of long-term adverse and challenging consequences of treatment, including immunosuppressive conditions; graftvs.-host disease (GvHD); other disorders related to chemotherapy toxicities; pulmonary, cardiovascular, and renal complications; chronic severe fatigue; and sexual dysfunction [15-20]. A number of studies indicate that at least $40 \%$ of HSCT survivors suffer from serious sequelae of the treatment that may last years and many long-term survivors report poorer health and increased health problems than healthy controls [21-25]. 
Such consequences of both disease and treatment present existential challenges to HSCT survivors: patients live with the threat of disease and frequently suffer from health complaints serious enough to affect a broad range of everyday activities, work and financial status, and social and intimate relationships [23, 24, 26-30]. Health-related quality of life (HRQoL), defined as an individual's satisfaction or happiness with domains of life insofar as they are affected by health [31], improves substantially in the first years following HSCT but often remains impaired [21, 32].

Given these long-term challenges, it is important to investigate whether psychosocial interventions, complementary to medical treatment, can be employed to enhance HRQoL and other dimensions of patient well-being. A number of studies have investigated effects of a peri- or posttreatment exercise program with promising effects [33]. However, very little research is available about psychosocial interventions for depression, fatigue and pain among HSCT survivors [34-36], although a substantial literature regarding psychosocial interventions exists for cancer patients [15, 37]. This is despite literature that documents persistent malaise among HSCT patients and suggests that pre-transplant parameters of well-being may be predictive of long-term physical and psychological outcome [38-41].

One recent, well controlled, randomized trial [36], among HSCT survivors, examined effects of a telephone-administered cognitive-behavioral intervention for post-traumatic stress disorder (PTSD) symptoms, as well as other measures of distress. Results indicated moderately positive benefits on interview measures of PTSD and general distress.

\section{Patients and methods}

\section{Patients}

This investigation was approved by the Ethics Commission of Both Basels. All patients were verified for the following inclusion criteria: (1) time since HSCT completion 6 months or longer, (2) complete remission of disease at time of enrollment, (3) no active malignancy-oriented therapy undertaken within the last 6 months, and (4) the patient evaluated as capable of participation by the attending medical hematologist. Excluded were patients with evidence of suicidality, reported or medically recorded diagnoses of current serious psychological disorders other than depression and anxiety or the inability to speak or read German. Patient characteristics are presented in Table 1.

Sixty-two HSCT patients were recruited for study participation after having received information via the outpatient clinic of the University Hospital Basel. Patients were contacted by a study nurse and provided with a brochure that
Table 1 Participant characteristics

\begin{tabular}{ll}
\hline Age (yrs) & 52.1 (SD, 14.1) \\
Gender (female, \%) & 50.0 \\
Time since transplant (years) & 7.5 (SD, 5.7) \\
Disorder (\%) & 21.0 \\
$\quad$ Non-Hodgkin's lymphoma & 17.7 \\
Acute lymphoblastic leukemia & 16.1 \\
Plasma cell disorder & 11.3 \\
Chronic myeloid leukemia & 11.3 \\
Acute myelogenous leukemia & 6.5 \\
Hodgkin's disease & 4.8 \\
Myelodysplastic syndrome & 4.8 \\
Chronic lymphocytic leukemia & 3.2 \\
Aplastic anemia & 1.6 \\
Autoimmune disease & \\
Transplant type (\%) & 30.6 \\
Autologous & 69.4 \\
Allogeneic & 25.6, Stage 0 \\
Chronic GvHD staging of recipients of allogeneic & 39.5, Stage 1 \\
HSCT (\%) & 34.9, Stage 2 \\
& \\
\hline
\end{tabular}

briefly described the program, mentioned its apparent efficacy in improving well-being among other patient groups, and stated the aim to evaluate the intervention's efficacy for enhancing well-being among long-term HSCT patients. The study was conducted from May 2010 to March 2012.

\section{Study protocols}

The original study design, analysis protocol, and documentation of all modifications are available from the first author. The study was conceived and began as a randomized controlled trial (RCT); almost exactly the first half of participants underwent RCT assignment. However, a number of complaints were lodged from patients while being recruited, as well as after having been randomized to their nonpreferred intervention: during recruitment, many patients reported their unwillingness to be assigned to the nonpreferred treatment program. One patient, allocated to the mindfulness group, but who would have preferred the control intervention, aired discontent about random assignment and manifested behavior thought by the instructor to be disruptive to group dynamics. These issues suggested that the RCT protocol was compromising the validity of the study (see "Discussion" for details). Therefore, a decision was made to continue the trial employing patient preference (PP) for the second half of the patient sample. Each assignment method is described below. 
Protocol designs

\section{Randomization}

Randomization for the first group of patients was conducted blindly by the principal investigator (PI). A pseudo-random process was be generated by an established online randomevent generator (www.randomizer.org). To maintain concealment of allocation, the following procedure was applied: all patients who met the inclusion criteria, first signed informed consent and completed all baseline assessments before they were then designated a sequential participation number by the PI who had no previous information about or contact with the patients. This list was then sent to the study coordinator who informed patients, by writing, of their assignment either to the mindfulness-based intervention (MBI) or the control, augmented optimal medical care program (AOMC). The process was controlled by the PI to ensure no violations of assignment.

\section{Patient preference protocol}

After determination of eligibility by one of the HSCT outpatient physicians, participants in the second half of the study were offered the intervention of their choice. All participants stipulated a preference. Duration of the intervention was 8 weeks.

All outcome measurements occurred within 2 weeks before the intervention (t1), within 2 weeks directly after the intervention (t2), and 3 months post-intervention (t3).

Assessments of all primary outcome variables were based upon patient-reported measures completed at home and returned by mail to the study coordinator. Data were entered blind to group assignment and checked by a person unfamiliar with the study hypotheses or group assignments of patients in order to verify accuracy. Therefore, data reported by the patients were not subject to biases of investigator or attending physician.

\section{Intervention}

MBI is based upon concepts of mental training that propose that mindfulness, defined as nonjudgmental awareness of moment-to-moment experience, may positively affect acceptance of intractable health-related changes, appreciation of available life experiences, realistic sense of control and accuracy of perception [42].

MBI was closely based upon the program of mindfulnessbased stress reduction (MBSR; [43, 44]) and included (1) a pre-intervention personal interview to define realistic goals of participants and establish personal rapport; (2) eight weekly 2.5 -h classes in mindfulness practices ( $\leq 15$ participants/group;
3) one Saturday, 6-h session at week 6; (4) homework assignments (approximately $40 \mathrm{~min} /$ day) emphasized as essential to success of the program; (5) a post-intervention interview to evaluate personal experiences, goal-attainment, and future maintenance of acquired skills; and (6) two 2.5-h booster sessions (end of months 1 and 2) of the 3-month follow-up phase. Each class covered specific exercises and topics within the context of mindfulness training, i.e., practices during lying, sitting, and dynamic yoga postures as well as everyday life, stressful situations, and social interactions (see [43] for details). Mindfulness exercises were aimed at intentional moment-to-moment awareness of sensory, affective, cognitive, and attitudinal domains of perceptible experience, while attempting, as well as possible, an attitude of kindness and openness to the immediate experience. The all-day retreat included already practiced exercises and new ones. In all, four MBI courses for the experimental arm of the study were individually conducted by three experienced, certified teachers, each with $>4$ years teaching experience.

Control group procedure: augmented optimal medical care (AOMC)

Patients in the control group (AOMC) received (a) regular, currently optimal care during the duration of the study, as provided by our HSCT outpatient department at the University Hospital Basel (also provided to the MBI group) and (b) 1530 min psychosocial consultations by telephone twice per month during the active 8-week intervention phase, administered by a clinical psycho-oncologist. Participants assigned to AOMC agreed upon dates and times for four individual telephone consultations over the 8-week intervention period. A structured telephone consultation was developed, guided by patients' needs and clinical experience. It treated topics that included changes in condition, family and emotional concerns, self-care, and other information about relevant aspects of psychosocial health specific to the patient and his or her family. During the follow-up period, patients had two additional telephone consultations, at the ends of month 1 and month 2 .

Recent evidence suggests that telephone consultations are well accepted by cancer patients and may provide a reasonable degree of social support without excessive effort on the part of patients [36, 45-48]. Employing this procedure in the control group of the current investigation may, therefore, control for some aspects of social support, as well as provide information about any supplemental efficacy of MBI.

Treatment fidelity was confirmed by regular consultations with all intervention teachers. There were no reported deviations from the intervention protocols. 


\section{Measures}

All measures were validated patient-reported outcomes, administered at pre-intervention, post-intervention, and 3-month follow-up. The primary outcome was HRQoL change at postintervention; depression, anxiety, and fatigue were secondary measures. Additionally, perceived personal goal attainment after MBI, and self-reported homework compliance were also assessed. Reported outcome measurements occurred within 2 weeks before and after intervention and 3-months postintervention.

The following instruments were used:

HRQoL was assessed with two inventories, one developed for patients with any of a broad range of chronic disorder (i.e., disease-aspecific) and the other developed specifically for cancer patients. The German-language Profile of Health-Related Quality of Life in Chronic Disorders (PQoLC) [49] was employed as the diseaseaspecific measure (six subscales: functional status, ability to relax and enjoy life, negative affect, positive affect, social functioning, and sense of belonging. Unlike most HRQoL measures that exclusively focus upon impairment, [50] this inventory includes a number of subscales of potentially positive areas of functioning. It has been well-validated in German-speaking populations, has excellent psychometric properties, and shows high sensitivity to change [44, 49, 51].

Disease-specific HRQoL was assessed with the Functional Assessment of Cancer Therapy Scale (FACT-G [52, 53], which contains five scales measuring physical, functional, social, and emotional well-being as well as satisfaction with the treatment relationship.

According to the a priori analysis plan in order to conserve statistical power, composite averages of subscales of each of the two HRQOL inventories were calculated, standardized, and normalized to a 5-point scale (0-4), which was then employed as primary outcome.

Depression, fatigue, and anxiety. Depression was measured with the Center for Epidemiological Studies Depression Scale (CES-D; [54, 55]; fatigue, with the Fatigue Questionnaire (FQ) [56]; and the FACIT-F fatigue scale [52] and anxiety, with the Spielberger Trait Anxiety Scale (STAI).

Goal Attainment Scale. At the post-intervention interview, MBI participants completed a goal attainment questionnaire that assessed the degree to which one-to-three pre-intervention personal goals had been achieved on an 11-point scale ( -5 [completely unmet] to +5 [far beyond expectation], with midpoint indicating goal attained to satisfaction. Goals varied from general (e.g., coping better) to specific (e.g., sleeping better). Data are presented as mean scores averaged across goals.
MBI participants also completed a questionnaire at follow-up regarding adherence to type, frequency, and duration of homework exercises performed during the previous 2 weeks.

\section{Statistical analyses}

The a priori power analyses for a comparison between the randomized groups estimated a minimal requisite sample size of 26 patients per group in order to detect a strong postintervention HRQoL effect size $(d=0.8$; see (44]) with a power of 0.80 and a two-tailed $\alpha$ of 0.05 . We originally planned on a sample size of 50 patients per group and intended to employ the depression scale as additional primary outcome. However, given that many patients often lived too far from the hospital to attend weekly MBI sessions, our original recruitment goal of 100 patients could not be met. Depression was then relegated to a secondary outcome.

Intention-to-treat (ITT) analyses are reported. Data of missing study patients were imputed by linear multiple regression that adjusted for age, gender, and pre-intervention score (STATISTICA 6.0). Repeated-measures group (MBI vs. AOMC) by time-point (post-intervention and follow-up) analyses of covariance (ANCOVA's) of change scores from preintervention levels, with pre-intervention score serving as covariate, were performed for each outcome. Preliminary analyses with a second grouping factor, randomized vs. PP groups, were performed for post-intervention. There were no significant differences or interaction effects with type of intervention, ( $p$ 's $>0.4$ for all outcomes), indicating that change of protocol did not affect overall findings regarding differences between treatments. Cohen's $d$-statistic [57] was employed as an estimate of effect size.

\section{Results}

Sample selection and attrition Study nurses telephoned 256 HSCT survivors from the registry of the University Hospital Basel who met entry criteria and lived in sufficient proximity of the study location. A total of 62 patients (24\%) participated. The RCT protocol included 32 patients; the PP protocol included 30 patients. MBI completers attended at least 6 of 11 sessions (mean attendance, $83.4 \%$ of course sessions, $50 \%$ of the booster sessions). Attrition rate (noncompleters) was $14.5 \%$ (20\% MBI; $7 \%$ AOMC), although $91 \%$ of MBI patients completed the intervention (12\% lost to follow-up). Further details regarding assignments to different arms of the study are provided in the adapted CONSORT flowchart (Fig. 1). 


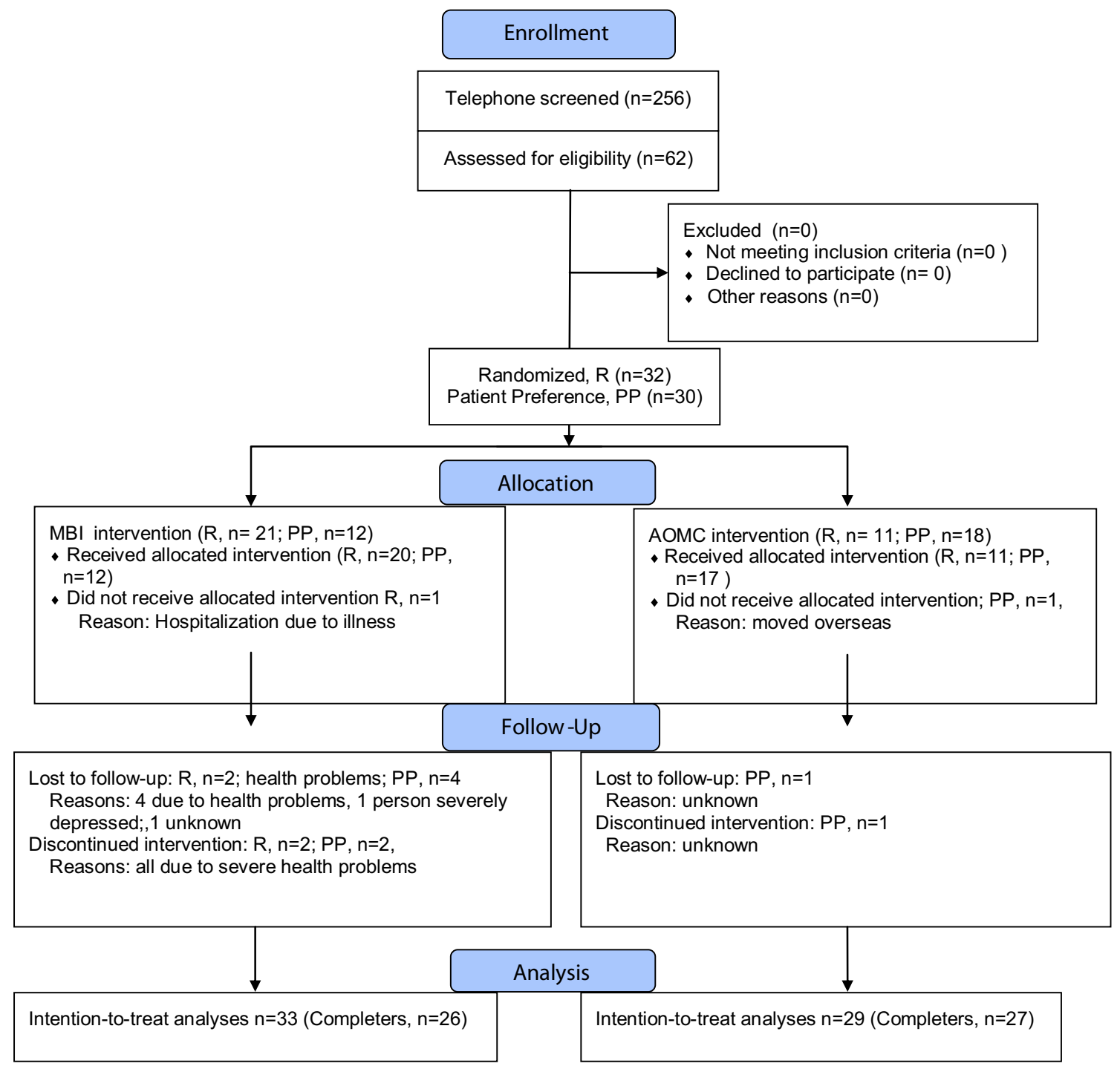

Fig. 1 Flowchart of the study

Differences between interventions Table 2 presents outcome data. Preliminary ITT ANCOVA's of post-treatment effects were performed with intervention (MBI vs. AOMC) and protocol type (RCT vs. PP) as grouping factor and preintervention level as covariate. Improvements were greater on HRQoL and depressive symptomology for the MBI group (respectively, $F$ 's $(1.57)=6.27$ and $6.04 ; p$ 's $<0.02$ ). Anxiety showed borderline significance in the same direction $(F$ $(1.57)=3.82 ; p<0.06)$. Fatigue did not improve between or across groups from pre- to post-intervention.

ANCOVA's of posttreatment and follow-up change scores showed significant group effects for HRQoL, depression, and anxiety. Fatigue did not improve with either intervention. No main effects of protocol type (RCT vs. PP) or intervention $\mathrm{X}$ protocol-type interactions were found for any outcome ( $F$ 's $(1.57)=0.00-0.68 ; p$ 's $=0.41-0.97)$; therefore, protocol type did not appear to influence overall results.
MBI goal attainment results The average goal attainment level was 1.66 (1.41, S.D.; range, -1.33 to 3.33 ), indicating generally greater perceived benefits than expected.

Reported adherence to home practice MBI patients reported practicing an average of 2.79 times/weeks (2.09, S.D.; median, 3 times/week) for a mean total of $89.10 \mathrm{~min}$ (86.56, S.D., median, $60 \mathrm{~min}$ ) per week.

\section{Discussion}

This investigation provides first evidence that mindfulness training may be effective at enhancing HRQoL and diminishing depressive symptoms and anxiety among HSCT survivors. In contrast to the AOMC, MBI improved post- 
Table 2 Means, standard deviations (SDs), and $95 \%$ confidence intervals (95\% CIs) on outcomes for patients before and after receiving MBI or AOMC

\begin{tabular}{|c|c|c|c|c|c|c|c|}
\hline & $\begin{array}{l}\text { Pre-intervention level } \\
\text { (mean, S.D.) }\end{array}$ & $\begin{array}{l}\text { Direct post-intervention } \\
\text { change }(95 \% \mathrm{CI})\end{array}$ & $\begin{array}{l}\text { 3-month follow-up } \\
\text { change }(95 \% \mathrm{CI})\end{array}$ & $F(1,59)$ & $P$ value & $\begin{array}{l}\text { Effect size } \\
\text { post }\end{array}$ & $\begin{array}{l}\text { Effect size } \\
\text { follow-up }\end{array}$ \\
\hline \multicolumn{8}{|l|}{ Outcome } \\
\hline \multicolumn{8}{|c|}{ HRQoL (range, 0-4) } \\
\hline $\operatorname{MBI}(n=33)$ & $2.57(0.58)$ & $0.18(0.07-0.28)$ & $0.15(0.04$ to 0.27$)$ & 4.50 & 0.04 & 0.68 & 0.27 \\
\hline $\operatorname{AOMC}(n=29)$ & $2.72(0.55)$ & $-0.06(-0.20-0.08)$ & $0.04(-0.10$ to 0.18$)$ & & & & \\
\hline \multicolumn{8}{|c|}{ CES-D (range, 0-60) } \\
\hline MBI & $15.45(10.62)$ & $2.56(-0.01-5.14)$ & $0.84(-1.14$ to 2.83$)$ & 4.55 & 0.04 & 0.70 & 0.19 \\
\hline AOMC & $13.70(9.07)$ & $-2.69(-5.62-0.23)$ & $-0.83(-3.82$ to 2.16$)$ & & & & \\
\hline \multicolumn{8}{|c|}{ STAI (range, 20-80) } \\
\hline MBI & $41.23(11.13)$ & $2.46(0.27-4.65)$ & $2.07(-0.17$ to 4.32$)$ & 4.37 & 0.04 & 0.58 & 0.37 \\
\hline AOMC & $39.18(10.59)$ & $-1.08(-3.21-1.05)$ & $-0.32(-2.32$ to 1.67$)$ & & & & \\
\hline \multicolumn{8}{|l|}{ FF (range, 0-52) } \\
\hline MBI & $33.23(9.56)$ & $-2.76(-5.10--0.42)$ & $-2.88(-5.17$ to -0.16$)$ & 1.31 & 0.26 & 0.01 & 0.02 \\
\hline AOMC & $34.24(9.27)$ & $-1.02(-3.72-1.68)$ & $-0.78(-3.53$ to 1.96$)$ & & & & \\
\hline
\end{tabular}

$P$ values indicate differences between groups in change from pre-intervention. Positive change always indicates improvement (e.g., increase in quality of life and decrease in depression or anxiety). Effect sizes=(differences of mean changes between groups) / (S.D. pooled pre-intervention)

MBI mindfulness-based intervention, AOMC augmented optimal medical care program, HRQoL health-related quality of life, CES-D Center for Epidemiological Studies Depression Scale, STAI Spielberger Trait Anxiety Inventory, FF FACT fatigue

intervention HRQoL and reduced depressive symptoms and anxiety. No benefits were found for fatigue. The positive effects were most pronounced at post-intervention and weakened at 3-month follow-up. Given that the booster sessions (which were only attended $50 \%$ of the time vs. $83 \%$ of the main MBI sessions) did not fully sustain benefits, these follow-up findings may, therefore, suggest that a longer term or perhaps continuing program of mindfulness intervention is needed to support HSCT survivors who suffer from chronic effects of past disease and sequelae of treatment.

We also addressed the question of what percentage of HSCT survivors might find supportive interventions helpful. One quarter of telephone-contacted patients were sufficiently motivated to take part in an intervention. Thus, it seems that a significant proportion of this clinical population may be interested to pursue interventions complementary to medical treatment for improving quality of life.

Our investigation additionally indicates the feasibility of MBI for patients who have undergone HSCT: the attrition rate was modest, especially given the amount of chronic health problems in this patient group. Four of the five patients who dropped out during the intervention phase did so because they became too ill to continue. Only $9 \%$ of the MBI group discontinued intervention (although another $11 \%$ were lost to follow-up). Furthermore, attendance of the program was good (mean of 9.4 of 11 sessions attended), and goal attainment results indicated general satisfaction with personal goals having been met.

Our change of protocol, not originally planned, requires comment. Particularly in behavioral intervention studies, and perhaps especially investigations with group interventions, $\mathrm{RCT}$ trials pose potential problems regarding ecological validity as well as the representativeness of findings to the general target population: under normal real-life circumstances, many people have preferences and actively choose the kind of behavioral intervention they want; in the real world, they are not randomly assigned to one treatment or another. Preferences are known to influence motivation and expectations; these may confound results in RCTs and influence group dynamics in intervention groups when one or more participants prefer to be in the alternative treatment arm. When patients with strong preferences consent to randomization, this is likely also affect its internal validity [58]: Those who receive preferred treatment are often more motivated, comply better with treatment, and report superior outcomes $[59,60]$. Patients not receiving a preferred treatment may experience demoralization, may be less motivated, may not comply with the treatment regimen, may not provide accurate self-report data during follow-up, and may even drop out of the study, contributing to further bias of the internal validity of a trial [61, 62].

These issues, therefore, pose conundrums for this study and behavioral intervention studies, in general. We attempted to address this problem, a posteriori, by applying a PP design to the second half of the study. We analyzed patients in the randomized part of the study and the patient-preference part of the study separately to detect differences but did not find any. However, the power to detect such differences was limited. For final analysis, patients in the randomized part of the study and those allocated by preference were pooled and analyzed by treatment received.

In conclusion, the results indicate that a mindfulness-based program is feasible and may increase HRQoL and reduce 
depression and anxiety among HSCT survivors. However, modest funding and scale of our study prevented us from using a control intervention with similar time commitment to MBI, as well as adjusting for potential confounders, e.g., disease severity, transplant type, and GVHD grade. Furthermore, effects seem unlikely to be sustained without some form of continued additional support. Therefore, a larger trial with a longer mindfulness intervention may be warranted to assess long-term benefits of MBI for HSCT survivors.

Acknowledgments We express deepest gratitude to all the patients who participated in this study, the mindfulness teachers Barbara DietzWaschkowski and Silvia Wiesmann as well to Silke Purschke and Susanne Müller, study nurses from the Clinical Trial Unit of our institution, who tirelessly recruited our participants. This study is dedicated to the memory of the investigation's lead MBI teacher Ulrike KesperGrossman (1953-2012).

\section{References}

1. Carlson LE, Doll R, Stephen J, Faris P, Tamagawa R, Drysdale E et al (2013) Randomized controlled trial of mindfulness-based cancer recovery versus supportive expressive group therapy for distressed survivors of breast cancer. J Clin Oncol 31(25):3119-3126

2. Hoffman CJ, Ersser SJ, Hopkinson JB, Nicholls PG, Harrington JE, Thomas PW (2012) Effectiveness of mindfulness-based stress reduction in mood, breast- and endocrine-related quality of life, and wellbeing in stage 0 to III breast cancer: a randomized, controlled trial. $\mathrm{J}$ Clin Oncol 30(12):1335-1342

3. Wurtzen H, Dalton SO, Elsass P, Sumbundu AD, Steding-Jensen M, Karlsen RV et al (2013) Mindfulness significantly reduces selfreported levels of anxiety and depression: results of a randomised controlled trial among 336 Danish women treated for stage I-III breast cancer. Eur J Cancer 49(6): 1365-1373

4. Carlson LE, Ursuliak Z, Goodey E, Angen M, Speca M (2001) The effects of a mindfulness meditation-based stress reduction program on mood and symptoms of stress in cancer outpatients: 6-month follow-up. Support Care Cancer 9(2):112-123

5. Speca M, Carlson LE, Goodey E, Angen M (2000) A randomized, wait-list controlled clinical trial: the effect of a mindfulness meditation-based stress reduction program on mood and symptoms of stress in cancer outpatients. Psychosom Med 62(5):613-622

6. Bauer-Wu S, Sullivan AM, Rosenbaum E, Ott MJ, Powell M, McLoughlin M et al (2008) Facing the challenges of hematopoietic stem cell transplantation with mindfulness meditation: a pilot study. Integr Cancer Ther 7(2):62-69

7. McArdle JR (2009) Critical care outcomes in the hematologic transplant recipient. Clin Chest Med 30(1):155-167

8. Michlitsch JG, Walters MC (2008) Recent advances in bone marrow transplantation in hemoglobinopathies. Curr Mol Med 8(7):675-689

9. Porta F, Locatelli F, Burgio GR (2008) Hematopoietic stem cell transplantation: 40 years of continuous progress and evolution. Haematologica 93(11):1607-1610

10. Petersdorf EW, Hansen JA (2008) New advances in hematopoietic cell transplantation. Curr Opin Hematol 15(6):549-554

11. Ferrara JL (2008) Advances in the clinical management of GVHD. Best Pract Res Clin Haematol 21(4):677-682

12. Gratwohl A (2002) New developments in hematopoietic stem cell transplantation. Ther Umsch 59(11):571-6

13. Passweg J, Baldomero H, Chapuis B, Leibundgut K, Schanz U, Gratwohl A (2006) Haematopoietic stem cell transplantation in
Switzerland. Report from the Swiss Transplant Working Group Blood and Marrow Transplantation (STABMT) Registry 1997 2003. Swiss Med Wkly 136(3-4):50-58

14. Carlson LE, Smith D, Russell J, Fibich C, Whittaker T (2006) Individualized exercise program for the treatment of severe fatigue in patients after allogeneic hematopoietic stem-cell transplant: a pilot study. Bone Marrow Transplant 37(10):945-954

15. Carr D, Goudas L, Lawrence D et al (2002) Management of cancer symptoms: pain, depression, and fatigue. Agency for Healthcare Research and Quality, Rockville, MD

16. Butt Z, Rosenbloom SK, Abernethy AP, Beaumont JL, Paul D, Hampton D et al (2008) Fatigue is the most important symptom for advanced cancer patients who have had chemotherapy. J Natl Compr Canc Netw 6(5):448-455

17. Syrjala KL, Kurland BF, Abrams JR, Sanders JE, Heiman JR (2008) Sexual function changes during the 5 years after high-dose treatment and hematopoietic cell transplantation for malignancy, with casematched controls at 5 years. Blood 111(3):989-996

18. Tichelli A, Rovo A, Gratwohl A (2008) Late pulmonary, cardiovascular, and renal complications after hematopoietic stem cell transplantation and recommended screening practices. Hematol Am Soc Hematol Educ Program 2008:125-133

19. Rovo A, Daikeler T, Stern M, Halter J, Studt JD, Buser A et al (2008) Physical and not mental health is impaired in very long-term survivors after HSCT compared with their respective donors: a paired analysis. Blood 111(3):1740-1741

20. Tichelli A, Bucher C, Rovo A, Stussi G, Stern M, Paulussen M et al (2007) Premature cardiovascular disease after allogeneic hematopoietic stem-cell transplantation. Blood 110(9):3463-3471

21. Hjermstad MJ, Knobel H, Brinch L, Fayers PM, Loge JH, Holte H et al (2004) A prospective study of health-related quality of life, fatigue, anxiety and depression 3-5 years after stem cell transplantation. Bone Marrow Transplant 34:257-266

22. Stein KD, Syrjala KL, Andrykowski MA (2008) Physical and psychological long-term and late effects of cancer. Cancer 112(11 Suppl):2577-2592

23. Bhatia S, Robison LL, Francisco L, Carter A, Liu Y, Grant M et al (2005) Late mortality in survivors of autologous hematopoietic-cell transplantation: report from the Bone Marrow Transplant Survivor Study. Blood 105(11):4215-4222

24. Bhatia S, Francisco L, Carter A, Sun CL, Baker KS, Gurney JG et al (2007) Late mortality after allogeneic hematopoietic cell transplantation and functional status of long-term survivors: report from the Bone Marrow Transplant Survivor Study. Blood 110(10):37843792

25. Khera N, Storer B, Flowers ME, Carpenter PA, Inamoto Y, Sandmaier BM et al (2012) Nonmalignant late effects and compromised functional status in survivors of hematopoietic cell transplantation. J Clin Oncol 30(1):71-77

26. Ness KK, Bhatia S, Baker KS, Francisco L, Carter A, Forman SJ et al (2005) Performance limitations and participation restrictions among childhood cancer survivors treated with hematopoietic stem cell transplantation: the bone marrow transplant survivor study. Arch Pediatr Adolesc Med 159(8):706-713

27. Norkin M, Hsu JW, Wingard JR (2012) Quality of life, social challenges, and psychosocial support for long-term survivors after allogeneic hematopoietic stem-cell transplantation. Semin Hematol 49(1):104-109

28. Shanis D, Merideth M, Pulanic TK, Savani BN, Battiwalla M, Stratton P (2012) Female long-term survivors after allogeneic hematopoietic stem cell transplantation: evaluation and management. Semin Hematol 49(1):83-93

29. Sun CL, Francisco L, Baker KS, Weisdorf DJ, Forman SJ, Bhatia S (2011) Adverse psychological outcomes in long-term survivors of hematopoietic cell transplantation: a report from the Bone Marrow Transplant Survivor Study (BMTSS). Blood 118(17):4723-4731 
30. Bevans MF, Mitchell SA, Barrett J, Bishop MR, Childs R, Fowler D et al (2013) Symptom distress predicts long-term health and wellbeing in allogeneic stem cell transplantation survivors. Biol Blood Marrow Transplant 20(3):387-395

31. Mitchell AJ, Benito-Leon J, Gonzalez JM, Rivera-Navarro J (2005) Quality of life and its assessment in multiple sclerosis: integrating physical and psychological components of wellbeing. Lancet Neurol 4(9):556-566

32. Mosher CE, Redd WH, Rini CM, Burkhalter JE, Duhamel KN (2008) Physical, psychological, and social sequelae following hematopoietic stem cell transplantation: a review of the literature. Psychooncology 18(2):113-127

33. Wiskemann J, Huber G (2008) Physical exercise as adjuvant therapy for patients undergoing hematopoietic stem cell transplantation. Bone Marrow Transplant 41(4):321-329

34. Kim SD, Kim HS (2005) Effects of a relaxation breathing exercise on anxiety, depression, and leukocyte in hemopoietic stem cell transplantation patients. Cancer Nurs 28(1):79-83

35. Kim SD, Kim HS (2005) Effects of a relaxation breathing exercise on fatigue in haemopoietic stem cell transplantation patients. J Clin Nurs 14:51-55

36. DuHamel KN, Mosher CE, Winkel G, Labay LE, Rini C, Meschian YM et al (2010) Randomized clinical trial of telephone-administered cognitive-behavioral therapy to reduce post-traumatic stress disorder and distress symptoms after hematopoietic stem-cell transplantation. J Clin Oncol 28(23):3754-3761

37. Mock V (2004) Evidence-based treatment for cancer-related fatigue. J Nat Cancer Inst Monographs 32:112-118

38. Hoodin F, Uberti JP, Lynch TJ, Steele P, Ratanatharathorn V (2006) Do negative or positive emotions differentially impact mortality after adult stem cell transplant? Bone Marrow Transplant 38(4):255-264

39. Akaho R, Sasaki T, Mori S-I, Akiyama H, Yoshino M, Hagiya K et al (2003) Psychological factors and survival after bone marrow transplantation in patients with leukemia. Psychiatry Clin Neurosci 57(1):91-96

40. Hochhausen N, Altmaier EM, McQuellon R, Davies SM, Papadopolous E, Carter S et al (2007) Social support, optimism, and self-efficacy predict physical and emotional well-being after bone marrow transplantation. J Psychosoc Oncol 25(1):87-101

41. Jenks Kettmann JD, Altmaier EM (2008) Social support and depression among bone marrow transplant patients. J Health Psychol 13(1): $39-46$

42. Grossman P, Niemann L, Schmidt S, Walach H (2004) Mindfulnessbased stress reduction and health benefits: a meta-analysis. J Psychosom Res 57(1):35-43

43. Kabat-Zinn J. Full Catastrophe Living: Using the Wisdom of Your Body and Mind to Face Stress, Pain and Illness. Revised Edition New York: Delacorte; 2013.

44. Grossman P, Kappos L, Gensicke H, D'Souza M, Mohr DC, Penner IK et al (2010) MS quality of life, depression, and fatigue improve after mindfulness training: a randomized trial. Neurology 75(13): 1141-1149

45. Beaver K, Tysver-Robinson D, Campbell M, Twomey M, Williamson S, Hindley A et al (2009) Comparing hospital and telephone follow-up after treatment for breast cancer: randomised equivalence trial. BMJ 338:a3147
46. Chamberlain Wilmoth M, Tulman L, Coleman EA, Stewart CB, Samarel N (2006) Women's perceptions of the effectiveness of telephone support and education on their adjustment to breast cancer. Oncol Nurs Forum 33(1):138-144

47. Chumbler NR, Mkanta WN, Richardson LC, Harris L, Darkins A, Kobb R et al (2007) Remote patient-provider communication and quality of life: empirical test of a dialogic model of cancer care. $\mathrm{J}$ Telemed Telecare 13(1):20-25

48. Coleman EA, Tulman L, Samarel N, Wilmoth MC, Rickel L, Rickel $\mathrm{M}$ et al (2005) The effect of telephone social support and education on adaptation to breast cancer during the year following diagnosis. Oncol Nurs Forum 32(4):822-829

49. Siegrist J, Broer M, Junge A (1996) Profil der Lebensqualität chronisch Kranker. Manual. [Profile of Quality of Life for the Chronically Ill]. Beltz Test, Göttingen

50. Hart S, Fonareva I, Merluzzi N, Mohr DC (2005) Treatment for depression and its relationship to improvement in quality of life and psychological well-being in multiple sclerosis patients. Qual Life Res 14(3):695-703

51. Grossman P, Tiefenthaler U, Raysz A, Kesper U (2007) Mindfulness training as an intervention for fibromyalgia: evidence of postintervention and 3-year follow-up benefits in well-being. Psychother Psychosom 76(4):226-233

52. Cella DF, Tulsky DS, Gray G, Sarafian B, Linn E, Bonomi A et al (1993) The functional assessment of cancer therapy scale: development and validation of the general measure. J Clin Oncol 11(3):570 579

53. Victorson D, Barocas J, Song J, Cella D (2008) Reliability across studies from the functional assessment of cancer therapy-general (FACT-G) and its subscales: a reliability generalization. Qual Life Res 17(9):1137-1146

54. Hautzinger M, Bailer M (1991) Allgemeine Depressionsskala (ADS). Die deutsche Version des CES-D. Weinheim, Beltz

55. Radloff LS (1977) The CES-D scale: a self-report depression scale for research in the general population. Appl Psychol Meas 1:385-401

56. Chalder T, Berelowitz G, Pawlikowska T, Watts L, Wessely S, Wright D et al (1993) Development of a fatigue scale. J Psychosom Res 37(2): 147-153

57. Cohen J (1988) Statistical power analysis for the behavioral sciences. Academic, New York

58. Schmoor C, Olschewski M, Schumacher M (1996) Randomized and non-randomized patients in clinical trials: experiences with comprehensive cohort studies. Stat Med 15:263-271

59. Lin P, Campbell DG, Chaney EF, Liu CF, Heagerty P, Felker BL et al (2005) The influence of patient preference on depression treatment in primary care. Ann Behav Med 30:164-173

60. Preference-Collaborative-Review-Group. Patients' preferences within randomised trials: systematic review and patient level meta-analysis. BMJ 2008;337:a1864

61. Howard L, Thornicroft G (2006) Patient preference randomised controlled trials in mental health research. Br J Psychiatry 188:303304

62. Torgerson DJ, Sibbald B (1998) Understanding controlled trials. What is a patient preference trial? BMJ $316: 360$ 